\title{
Recovering Recovery: On the relationship between gauge symmetry and Trautman recovery
}

\author{
Nicholas J. Teh \\ Department of Philosophy \\ Malloy Hall \\ University of Notre Dame
}

August 2, 2017

\begin{abstract}
This paper (i) uncovers a foundational relationship between the 'gauge symmetry' of a Newton-Cartan theory and the celebrated Trautman Recovery Theorem; and (ii) explores its implications for recent philosophical work on Newton-Cartan gravitation.
\end{abstract}

\section{Introduction}

The fortunes of Newton-Cartan theory are on the rise. Once regarded by physicists as a mere mathematical curiosity, the theory has enjoyed a renaissance within physics in recent years, stemming from two distinct but related sources, viz. the use of Newton-Cartan theory to describe condensed matter phenomena (especially the fractional Quantum Hall Effect), and the prospects of using Newton-Cartan theory to define a non-relativistic version of holographic duality ${ }^{1}$ Within philosophy, on the other hand, Newton-Cartan gravitation has long been a popular subject; but here too there has been much recent development, as we will soon discuss.

There are marked differences between these two traditions of applying Newton-Cartan theory. On the one hand, the physics literature is concerned with highly general Newton-Cartan backgrounds to which various types of

\footnotetext{
${ }^{1}$ For the condensed matter applications, see Geracie et al. (2015); and for the holographic applications, see Bergshoeff et al. (2015) and references therein.
} 
Copyright Philosophy of Science 2017

Preprint (not copyedited or formatted)

Please use DOI when citing or quoting

fields can be coupled; within this context, the gauge symmetry (i.e. the 'descriptive freedom') of the theory has been identified as 'Milne symmetry' (cf. (8)). By contrast, the philosophical literature has a more limited scope in that it only considers models of (Newtonian) gravitation; however, it also explores more foundational themes such as the empirical equivalence between a Newton-Cartan model and its 'recovered' set of Neo-Newtonian models, as articulated in the Trautman Recovery Theorem. This theorem informs us of a gauge symmetry (cf. (5)) that acts on the set of recovered models - call this 'Trautman symmetry'.

It would be of considerable interest to bring these two traditions into dialogue with each other, and it is an especially tantalizing thought that philosophy might be able to bring foundational discussions about 'empirical equivalence' to bear on cutting-edge developments in physics. The main results of this paper (Props. 4.3.1 and 4.4.1) pave the way for this endeavor by uncovering a systematic relationship between a trio of concepts, viz. 'Milne symmetry', 'Trautman symmetry', and 'recovery'. More specifically, we show that 'recovery' can be understood as the relationship between two different gauge-fixings of the general Milne gauge symmetry - this results in a powerful and informative characterization of the set of recovered models, which incorporates symmetry data that is implicit in the 'vector relationism' of Saunders (2013) ? $^{2}$

Our results also illuminate and synthesize some recent developments in the philosophy of Newton-Cartan gravitation. The relevant body of work originates in Saunders (2013) and Knox (2014), who provide different respective arguments for the thesis that Neo-Newtonian spacetime is not the correct setting for Newtonian gravitation. On the one hand, Saunders formulates Newtonian gravitation in a 'vector relationist' framework and uses this to argue that its correct spacetime setting is 'Maxwell spacetime', i.e. a spacetime equipped with a standard of rotation but no standard of linear acceleration. On the other hand, Knox focuses on the Trautman gauge symmetry of empirically equivalent Neo-Newtonian models, and argues that we should move to a gauge-invariant formulation of the theory's spacetime, i.e. Newton-Cartan spacetime.

While the subsequent literature can in a broad sense be understood as demonstrating the 'equivalence' between Maxwell gravitation and NewtonCartan gravitation, it will be helpful for us to divide it into two strands. The first strand, taken up by Weatherall (2016) and Dewar (2017), uses standard differential geometry (and Trautman Recovery) to argue that Newton-Cartan and Maxwell gravitation are equivalent in the sense that, given a model of

\footnotetext{
${ }^{2}$ We thank an anonymous reviewer for helping us to frame the point in this way.
} 
Copyright Philosophy of Science 2017

Preprint (not copyedited or formatted)

Please use DOI when citing or quoting

Newton-Cartan gravitation, one can define a unique model of Maxwell gravitation, and vice versa. The second strand, taken up by Wallace (2016), forges a direct link between Saunders's vector relationism and Wallace's own mathematically 'idiosyncratic' (because coordinate-transform-based) formulation of Newton-Cartan gravitation, thereby showing that the latter is equivalent to Maxwell gravitation.

One way, then, of summarizing our main contribution is that it provides an illuminating synthesis of these two strands. First, the 'representation theory' introduced in Section 3 shows that the physicist's notion of (Milne) gauge symmetry can be interpreted as a way of incorporating vector relationism into the standard differential geometric framework for Newton-Cartan gravitation. This thus alleviates some of Wallace's worries concerning the differential geometric framework, viz. that it obscures physical intuition and that it is an awkward tool for understanding a spacetime's 'standard of rotation'. Indeed, the Proto-recovery result of Section 4 not only exhibits an elegant and direct way of understanding 'the same standard of rotation' (viz. as representations that share the same vorticity), but also isolates the deep structural feature that gives rise to this 'sameness': at the level of curvature, it is the Newtonian condition; and at the level of symmetry, it is the invariance of the standard of rotation under a certain $U(1)$ symmetry acting on gauge fields (cf. Section 4.2). This point tends to be obscured by the first strand, which closely adheres to the strategy of the original Trautman Recovery result; by contrast, our reconceptualization of 'recovery' shows that the original Trautman result and recent results about Maxwell gravitation are most perspicuously understood as two aspects of a unified 'recovery' package. Finally, Section 5 discusses three more specific applications that flow from our results: the explanation of the Maxwell equations of motion given in Dewar (2017); a more fine-grained analysis of the analogy between Newton-Cartan gravitation and Maxwell gauge theory; and the possibility of a parallel geometrization/recovery result for vorticity (as opposed to linear acceleration).

\section{Background and notational preliminaries}

Let $M$ be a smooth $(n+1)$-dimensional manifold that is equipped with: $t_{a}$ a non-vanishing closed 1-form (the 'time metric') and $h^{a b}$ a rank $n$ positivesemidefinite symmetric tensor (the 'space metric') such that $h^{a b} t_{b}=0$. We will always assume that $M$ is simply connected, or that we are only interested in a contractible patch of $M$. A tangent vector $N$ is time-like just in case $t(N) \neq 0$, and it is spacelike just in case it is in the kernel of $t$. It is easy to see that a vector $V^{a}$ is spacelike iff it can be written as $h^{a b} \chi_{b}$ and we shall pass 
Copyright Philosophy of Science 2017

Preprint (not copyedited or formatted)

Please use DOI when citing or quoting

freely between these descriptions. We will also be at liberty to use index-free notation (e.g. $\left.h(d \phi)=h^{a b} \nabla_{b} \phi\right)$ when it simplifies our expressions.

The basic 'manifold with (degenerate) metric' structure of interest to us will be that of a Leibnizian geometry $\mathcal{L}$, i.e. the triple $(M, t, h)$. We shall at times be concerned with the question of what sorts of structures can be defined solely in terms of the Leibnizian structure: we shall call such structures 'canonical'.

At a purely kinematic level, the trajectories of observers/bodies in $\mathcal{L}$ can be described by a time-like unit vector field $N$, whose integral curves are the relevant trajectories. We shall denote the affine space of such fields of observers by $\mathcal{F}(M, t)$. Since the differences of such observer fields are spacelike vectors, $\mathcal{F}$ is modeled on the vector space $\Gamma(\operatorname{Ker} t)$ of spacelike vector fields, which can also be viewed as an additive group called the Milne group. The Milne group has a free and transitive Milne boost action on $\mathcal{F}$, i.e. $N \mapsto N+V$, where $N \in \mathcal{F}$ and $V \in \Gamma($ Ker $t)$.

We will often be concerned with tensorial objects that depend on a field of observers $N \in \mathcal{F}$. For instance, given $N$, we can define the transverse metric $h_{a b}$ by means of the relations $h_{a b} N^{b}=0$ and $h^{a b} h_{b c}=h_{c}^{a}:=\delta_{c}^{a}-N^{a} t_{c}$, where $h_{c}^{a}$ is an $N$-dependent transverse projector. We will use oversetting when we wish to highlight this dependence, e.g. $h_{a b}^{N}$.

The next level of structure that will be of interest to us is that of a 'geometry with connection'. We shall use the term Newton-Cartan connection to refer to a torsion-free connection $\nabla$ that is compatible with a Leibnizian geometry $\mathcal{L}$, i.e. a connection that satisfies $\nabla_{a} h^{b c}=0$ and $\nabla_{a} t_{b}=0$. Furthermore, we will primarily be concerned with Newton-Cartan connections that are Newtonian, meaning that they satisfy the curvature condition (R1) $R_{c d}^{a b}=R_{d c}^{b}{ }_{c}^{a}$. The Newtonian condition is equivalent to the closedness of a set of 2 -forms that will be defined in Section 3.2, and we will use only this formulation in what follows.

Given a field of observers $N$, it is convenient to analyze the physics of its integral curves in terms of three parameters, viz.

- The vorticity $\stackrel{N}{\omega}_{a b}^{N}:=h_{[a}^{m} h_{b] n}^{N} \nabla_{m} N^{n}$, which measures the rotation of an infinitesimal volume element under the flow of $N$. When it vanishes, we shall say that $N$ is twistless.

- The expansion $\stackrel{N}{\theta_{a b}}:=h_{h_{(a}^{m}}^{N} h_{b) n}^{N} \nabla_{m} N^{n}$. When it vanishes, we shall say that $N$ is rigid.

- The acceleration $\alpha^{b}:=N^{a} \nabla_{a} N^{b}$. When it vanishes, we shall say that $N$ is geodesic. 
Copyright Philosophy of Science 2017

Preprint (not copyedited or formatted)

Please use DOI when citing or quoting

When there are multiple connections in play, we will sometimes overset $\nabla$ to indicate which connection is being used in a parameter, e.g. $\stackrel{\nabla, N}{\omega}$.

The sorts of theories that we will consider all have models whose structure includes a 'Leibnizian geometry with a Newtonian connection'. Furthermore, these connections sometimes satisfy a second curvature condition (R2) $R_{c d}^{a b}=$ 0 . (R2) of course implies the weaker 'spatial flatness' condition (R2') $R^{a b c d}=$ 0 , which we will have reason to consider independently in Section 4.4.

Here are two gravitational models of interest to us:

1. Let $\nabla$ be a Newtonian connection that is flat, and thus satisfies (R2). Let $\rho$ and $\phi$ be scalar fields on $\mathcal{L}$ that represent the mass density and gravitational potential respectively, and let $N \in \mathcal{F}$ be a dynamical vector field that plays the role of a solution to the theory's equation of motion. A Neo-Newtonian model of gravitation is the structure $(\mathcal{L}, \nabla, \phi, \rho, N)$ that satisfies the following dynamical constraints, viz. the equation of motion

$$
\alpha^{a}=-\nabla^{a} \phi
$$

where $\alpha$ is the (spacelike) acceleration vector field; and the source equation

$$
\nabla_{a} \nabla^{a} \phi=4 \pi \rho .
$$

2. Let $\nabla$ be a curved Newtonian connection that satisfies (R2). A NewtonCartan model of gravitation is the structure $(\mathcal{L}, \nabla, \rho, N)$ that satisfies the following dynamical constraints, viz. the geodesic equation of motion

$$
\alpha^{a}=0
$$

and the 'geometrized' source equation

$$
R_{a b}=4 \pi \rho t_{a} t_{b}
$$

Note that the source equation implies (R2') ${ }^{3}$

We can now summarize more precisely the content of the Trautman Recovery Theorem: Given a Newton-Cartan model $(\mathcal{L}, \widetilde{\nabla}, \rho, N)$, one can reconstruct a Neo-Newtonian model $(\mathcal{L}, \nabla, \phi, \rho, N)$, up to what we have called the 'Trautman gauge symmetry':

$$
\nabla \mapsto \nabla^{\prime}=\left(\nabla, t_{b} t_{c} \nabla^{a} \psi\right), \quad \phi \mapsto \phi^{\prime}=\phi+\psi
$$

where the scalar field shift $\psi$ is required to satisfy

$$
\nabla^{a} \nabla^{b} \psi=0
$$

\footnotetext{
${ }^{3}$ For a proof of this statement, see Prop. 4.1 .5 of Malament (2012).
} 
Copyright Philosophy of Science 2017

Preprint (not copyedited or formatted)

Please use DOI when citing or quoting

In other words, the Trautman symmetry maps $(\mathcal{L}, \nabla, \phi, \rho, N)$ to $\left(\mathcal{L}, \nabla^{\prime}, \phi^{\prime}, \rho, N\right)$, and the theorem tells us that an entire Trautman symmetry orbit can be recovered from a single Newton-Cartan model.

For ease of reading, we have included proof sketches of only our main results (Props. 4.3.1 and 4.4.1) in the main body of the text. The references or proofs for all other propositions have been relegated to Appendix A.

\section{Symmetry and invariance from a repre- sentational strategy}

\subsection{Representing Newton-Cartan connections}

In the physics literature, it is a commonplace that a Newton-Cartan connection can be specified by means of an observer vector field $N$ and a 2 -form $F$, at least up to 'Milne gauge transformations'. But while this fact is useful for computations, it is hardly illuminating; and moreover, it is difficult to see what its relationship might be (if any) to what philosophers refer to as the 'gauge symmetry' of Neo-Newtonian models 4 We must thus seek out a more incisive analysis of this gauge symmetry if we are to achieve our end; we claim that such an analysis will stem from the recent work of Bekaert and Morand (2016) (henceforth BM) on 'representing' a Newton-Cartan connection.

BM note that although the requirement of compatibility with a Leibnizian geometry does not determine a unique Newton-Cartan connection (cf. the Levi-Civita connection), there is still room for an analogy between NewtonCartan connections and torsionful Lorentzian connections: for the latter, too, are not uniquely determined by the requirement of compatibility. On this basis, they argue that a familiar strategy for representing torsionful Lorentzian connections can be transferred to the case of Newton-Cartan connections.

Their 'physically perspicuous' representational strategy takes the following form: First, notice that the affine space $\mathcal{C}$ of Newton-Cartan connections is modeled on a vector space $\mathcal{V}$ (whose vectors are differences between connections), which is in turn canonically isomorphic to the vector space $\Omega^{2}$ of 2 -forms on $M$. We can thus view $\mathcal{C}$ as an affine space that is modeled on $\Omega^{2}$.

Next, endow $\mathcal{C}$ with a choice of origin (call the resulting vector space $\mathcal{C}_{0}$ ) by means of the following vector space isomorphism:

$$
\stackrel{N}{\Theta}: \mathcal{C}_{0} \rightarrow \Omega^{2}, \quad \nabla \mapsto \stackrel{N}{F}_{a b}:=-2 \stackrel{N}{h}_{c[a} \nabla_{b]} N^{c} \quad \text { where } \quad N \in \mathcal{F}
$$

\footnotetext{
${ }^{4}$ See e.g. Knox (2014) for this use of 'gauge symmetry'.
} 
Copyright Philosophy of Science 2017

Preprint (not copyedited or formatted)

Please use DOI when citing or quoting

Evidently, the origin or 'reference point' is $\Theta^{-1}(0)$, i.e. it is the connection such that $\stackrel{N}{F} \in \Omega^{2}$ vanishes. The Newton-Cartan literature dignifies such reference connections by means of the following definition: A (Newton-Cartan) connection is special just in case for some $N \in \mathcal{F}, \stackrel{N}{F}$ vanishes with respect to that connection. We denote a special connection by $\stackrel{N}{\nabla}$, which also helps us keep track of relevant observer vector field $N$.

Having made this choice of origin, it follows that an arbitrary NewtonCartan connection $\nabla \in \mathcal{C}$ can be uniquely specified as a vector from the origin. We shall thus say that the pair $(\stackrel{N}{\nabla}, \stackrel{N}{F})$ represents $\nabla$ by providing (i) the reference point data $\stackrel{N}{\nabla}$ with respect to which $\nabla$ is being represented; and (ii) the directional data $\stackrel{N}{F}$ that unambiguously picks $\nabla$ out with respect to the reference point. In fact, we obtain a slightly more efficient formulation if we recall a classical result, viz. that for any $N \in \mathcal{F}$, there exists a unique special connection $\stackrel{N}{\nabla}$; the representation can then be expressed as $(N, \stackrel{N}{F}) .5$

Why should this representational strategy be deemed physically perspicuous? To see this, note first that the directional data $\stackrel{N}{F}$ has a physical interpretation: its physical content consists of the (linear) acceleration $\stackrel{N}{\alpha}$ and the vorticity $\stackrel{N}{\omega}$ experienced by a field of observers $N \sqrt[6]{6}$ To remind ourselves of this fact, we will call $\stackrel{N}{F}$ a Newton-Coriolis 2-form, and $(N, \stackrel{N}{F})$ a Newton-Coriolis representation of $\nabla$.

Next, we consider the physical interpretation of the reference point $\stackrel{N}{\nabla}$ relative to the directional data. Since $\stackrel{N}{\nabla}$ is special, it can be represented by $(N, 0)$. It thus follows from the preceding discussion that $N$ is geodesic and twistless with respect to $\stackrel{N}{\nabla}$. In other words, $\stackrel{N}{\nabla}$ can be interpreted as encoding a 'generalized inertial structure' (call this 'inertial*') with respect to which $N$ is the vector field corresponding to inertial* trajectories. Thus, Newton-Coriolis representations are physically perspicuous because their reference point data provides a background inertial* structure with respect to which further physicsi.e. the forces encoded in a non-vanishing Newton-Coriolis 2-form-can be described; furthermore, by specifying such forces, we can pick out an arbi-

\footnotetext{
${ }^{5}$ See e.g. Prop. 3.9 of Bekaert and Morand (2016) and references therein.

${ }^{6}$ More precisely, these physical parameters can be extracted from the relations $\stackrel{N}{F}(N, V)=$ $\stackrel{N}{h}(\stackrel{N}{\alpha}, V)$ and $\stackrel{N}{F}(V, W)=\stackrel{N}{\omega}(V, W)$, where $N \in \mathcal{F}$, and $V$ and $W$ are spacelike vector fields.
} 
Copyright Philosophy of Science 2017

Preprint (not copyedited or formatted)

Please use DOI when citing or quoting

trary Newton-Cartan connection. This insight also gives us the resources to understand how Newton-Coriolis representations implicitly encode Saunders's 'vector relationist dynamics'. As articulated by Wallace (2016), vector relationism can be divided into two components: (i) test particles that define idealized background inertial structures (and relative accelerations between pairs of test particles, cf. eqns (56-58) of (Wallace, 2016)); and (ii) motions of particles relative to the test particles. But the vector field of the trajectories of a test particle is nothing other than some $N \in \mathcal{F}$, and so it is evident that the background inertial structure of (i) is encoded in the reference point data $\stackrel{N}{\nabla}$, with respect to which the directional data corresponds to part (ii) of Wallace's schema. As a special case, the relative acceleration between two inertial* structures $\stackrel{N}{\nabla}$ and $\stackrel{N^{\prime}}{\nabla}$ can be expressed as $\stackrel{N}{F}$, i.e. the directional data of $\stackrel{N^{\prime}}{\nabla}$ when it is represented by $(N, \stackrel{N}{F})$.

We now consider the sense in which the above representational strategy provides a perspicuous account of how the physicist's 'Milne gauge symmetry' arises, and an incisive notion of 'gauge choice' with respect to this symmetry. The root of this descriptive freedom can be found in the fact that the isomorphism (7) manifestly depends on data over above the Leibnizian geometry, viz. a choice of $N$. It is thus clear that there is a whole family $\{\stackrel{N}{\nabla}\}_{N \in \mathcal{F}}$ of reference connections which provide distinct-but equally good-representations of a Newton-Cartan connection.

What is the relationship between these representations? The answer is evident when we recall the Milne boost $N \mapsto N^{\prime}=N+V$ of Section 2, which in turn induces the following Milne symmetry action on the space of NewtonCoriolis representations, i.e.

$$
(N, \stackrel{N}{F}) \mapsto\left(N^{\prime}, \stackrel{N^{\prime}}{F}=\stackrel{N}{F}+d \stackrel{N, V}{\Phi}\right), \quad \text { where } \stackrel{N, V}{\Phi}=\stackrel{N}{\Phi_{c b}} V^{b}-\frac{1}{2} \stackrel{N}{h}{ }_{a b} V^{a} V^{b} t_{c}
$$

This is of course nothing other than what we referred to earlier as the physicist's 'Milne gauge symmetry'. However, our intellectual path has given us a much deeper understanding of this concept, viz. that it is underwritten by the above representational strategy, and that a gauge choice (with respect to the Milne symmetry) is given by the representation $(N, \stackrel{N}{F})$. As we shall soon see, the fact that a gauge choice refers to the reference data $\stackrel{N}{\nabla}$ will be crucial to our argument.

We are now in a position to discuss the invariance of the connection with respect to the Milne gauge symmetry, and the sense in which this provides a 'standard of sameness' for Newton-Cartan connections. First, note that 
Copyright Philosophy of Science 2017

Preprint (not copyedited or formatted)

Please use DOI when citing or quoting

the connection is a well-defined object in its own right; it does not need to be described using the notion of Milne symmetry. However, given that we have adopted a descriptive scheme in which many different gauge choices $\{(N, \stackrel{N}{F})\}_{N \in \mathcal{F}}$ can be used to pick out the same connection, the question arises as to how the Milne symmetry can be used to describe the connection invariantly, i.e. in a manner that is independent of any particular choice of gauge. As one might expect, this can be done by identifying a Newton-Cartan connection with the Milne orbit (i.e. gauge equivalence class) of one of its Newton-Coriolis representations (i.e. gauge choices). That such an identification makes sense is the content of the following proposition:

Proposition 3.1.1. The affine space of Milne orbits in $\mathcal{F} \times \Omega^{2}$ is canonically isomorphic to the affine space of Newton-Cartan connections.

We thus have a Milne symmetry-based standard by which to judge that a set of models possesses a certain connection structure, viz. we require that the set of models has sufficient data to define the Milne orbit $[(N, F)]$ corresponding to that connection.

\subsection{Representing Newtonian connections}

The models of interest in this paper all use Newtonian connections, i.e. connections whose Newton-Coriolis 2 -forms are closed 7 Thus, given a Newtonian connection $\nabla$, the space of its Newton-Coriolis representations is $\mathcal{F} \times \Omega_{\text {closed }}^{2}$ and the invariant content of a Newtonian connection can be identified with the corresponding Milne orbit in this space. (Note that since, locally, a connection is Newtonian just in case it is special, the same point applies to special connections 8

Let us emphasize that this method of imposing the Newtonian condition does not change anything about the observer vector fields over which we quantify in order to describe different representations of the connection. Rather, it changes the structure of the directional data $\stackrel{N}{F}$ that we use to pick out the connection. We now explore the implications of this change.

From Poincaré's Lemma, we know that any closed form is locally an exact form. Thus, given a Newtonian connection, each of its Newton-Coriolis 2-

\footnotetext{
${ }^{7}$ See e.g. Künzle $(1972)$ for a proof that this definition of the Newtonian condition is equivalent to the definition in terms of the Riemann tensor.

${ }^{8}$ The local equivalence of Newtonian connections and special connections is well-known to experts and has appeared several times in the literature. See e.g. Props. 3.18 and 3.26 of BM and Props. 4.3.4 and 4.3.7 of Malament (2012).
} 
Copyright Philosophy of Science 2017

Preprint (not copyedited or formatted)

Please use DOI when citing or quoting

forms $\stackrel{N}{F}$ has a local description as the 'gauge field' $\stackrel{N}{A}$, where $\stackrel{N}{A}$ is a 1-form such that $\stackrel{N}{F}=d \stackrel{N}{A}$. It will also be clear from the gauge theory analogy that $\stackrel{N}{F}$ does not correspond to a single gauge field $\stackrel{N}{A}$, but rather to the equivalence class $N$

$[A]$ whose equivalence relation is given by the Maxwell gauge transformation $A \mapsto A+d f$, where $f$ is an arbitrary 0 -form. We will call $(N,[\stackrel{N}{A}])$ a principal connection representation, and we will call each of its Maxwell representatives $(N, \stackrel{N}{A})$ a gauge field representation.

Evidently, using a gauge field representation in place of a Newton-Coriolis representation allows us to describe the structure of $\nabla$ in a way that increases the gauge symmetry acting on the directional data of a representation. On the one hand, the Milne symmetry (cf. (8)) acts on principal connection representations as follows

$$
(N,[\stackrel{N}{A}]) \mapsto(N+V,[\stackrel{N}{A}+\stackrel{N, V}{\Phi}]),
$$

where $\Phi$ is defined in (8). And on the other hand, each representation carries within it the action of the Maxwell gauge symmetry, as indicated by the Maxwell orbit $[A]$. Thus, an orbit of $[9]$ is the equivalence class $[(N,[A])]$, where $(N, \stackrel{N}{A}) \sim\left(N^{\prime}, \stackrel{N^{\prime}}{A}\right)$ just in case $N^{\prime}=N+V$ and $\stackrel{N^{\prime}}{A}=\stackrel{N}{A}+\stackrel{N, V}{\Phi}+d f$; let us call this a Milne-Maxwell orbit. By similar reasoning to that used in Prop. 3.1.1, one can show:

Proposition 3.2.1. The affine space of Milne-Maxwell orbits is canonically isomorphic to the affine space of Newtonian connections.

We will need one last important fact about gauge field representations, viz. that they afford us a way of constructing Milne-invariant quantities in terms of the gauge fields $\stackrel{N}{A}$. As is well-known in the literature, there are three such quantities: a scalar, a vector, and a metric Milne-invariant respectively 9

$$
\begin{gathered}
\phi:=2 \stackrel{N}{A_{b}}\left(N^{b}\right)-h^{a b} \stackrel{N}{A_{a} A_{b}} \\
Z^{a}:=N^{a}-h^{a b} \stackrel{N}{A_{b}} \\
g_{a b}:={ }_{h}^{N}+2 t_{(a} \stackrel{N}{\left.A_{b}\right)} .
\end{gathered}
$$

\footnotetext{
${ }^{9}$ More precisely, there are three such quantities up to Maxwell symmetry transformations - we address this point for $\phi$ and $Z$ in the next section, and the analogous point for $g$ is addressed in Section 3.4 of BM.
} 
Copyright Philosophy of Science 2017

Preprint (not copyedited or formatted)

Please use DOI when citing or quoting

These quantities are Milne-invariant in the sense that they remain unchanged under the action of the Milne symmetry on $N, \stackrel{N}{A}$, and $\stackrel{N}{h}$, i.e. the $N$-dependent quantities in terms of which they are exclusively defined (over above the Leibnizian structure). Notice that $Z$ is a vector field of observers, and that $g$ is non-degenerate 10 Notice further that the invariants can be defined in terms of each other and the Leibnizian structure, e.g. $g_{a b} Z^{b}=t_{a} \phi$. While these Milne-invariants are interesting for a variety of reasons, their primary interest for us is that they will allow us to construct a third representation: one that will finally let us make contact with the Trautman Recovery Theorem. In the next section, we use the insights that we have developed about 'gauge' to pursue this goal.

\section{Recovery from gauge symmetry}

\subsection{Motivating Proto-recovery}

The Trautman Recovery Theorem assumes that its (curved) Newton-Cartan models involve a Newtonian connection $\nabla$ that satisfies the curvature condition (R2). It is furthermore easy to see that (R2-Equiv): (R2) holds of $\nabla$ iff (R2') holds of $\nabla$ and there exists a twistless and rigid vector field of observers $N \in \mathcal{F}$ with respect to $\nabla$, i.e. $\stackrel{N}{\omega}=\stackrel{N}{\theta}=0.11$

In light of this equivalence, a well-known result called the Künzle-Ehlers Recovery Theorem proceeds to generalize Trautman Recovery by dropping the twistlessness condition on such a vector field, i.e. it only requires the existence of a rigid vector field of observers ${ }^{12}$ And since a rigid vector field of observers exists just in case (R2') holds, this generalization is tantamount to replacing the stronger curvature condition (R2) with the weaker spatial flatness condition $\left(\mathrm{R} 2{ }^{\prime}\right)$ 13

We remind the reader of these classical results because we wish to highlight the fact that there exists a complementary, albeit previously unexplored, direction of generalization to the one pursued by Künzle-Ehlers Recovery, i.e. where one drops rigidity and retains twistlessness instead of dropping twistlessness and retaining rigidity. Might it be possible to obtain a different generalized recovery result (from Künzle-Ehlers) if we required only the existence of a

\footnotetext{
${ }^{10}$ More precisely, $g$ is non-degenerate when $\phi$ is nowhere vanishing.

${ }^{11}$ See e.g. Prop. 4.2.4 of Malament (2012).

${ }^{12}$ We refer the reader to Section 4.5 of Malament (2012) for an account of the Künzle-Ehlers Recovery Theorem.

${ }^{13}$ For a proof of the first part of this statement, see Prop. 4.5.1 of Malament (2012).
} 
Copyright Philosophy of Science 2017

Preprint (not copyedited or formatted)

Please use DOI when citing or quoting

twistless vector field of observers? It turns out that it is indeed possible to prove such a result, which we call 'Proto-recovery' in order to highlight its more general and highly schematic nature. Furthermore, reconceiving Trautman Recovery as a special case of Proto-recovery will be precisely what is needed to illuminate the relationship between Milne symmetry and Trautman symmetry.

As we have just seen, facts linking curvature conditions to the existence of vector fields satisfying certain physical properties are crucial to understanding the Trautman and Künzle-Ehlers recovery results. Thus, in order to obtain our desired generalization, we will need to understand a similar relationship that involves only twistlessness. This comes in the form of (R1-Equiv): $\nabla$ is Newtonian just in case there exists a family of twistless (with respect to $\nabla)$ vector fields whose associated Newton-Coriolis 2 -forms are closed. We now turn to an explanation of this statement and its relationship with the notion of 'gauge choice' developed in Section 3.

\subsection{Twistless gauge-fixing and the Maxwell repre- sentation}

Recall that in Section 3.2, the Newtonian condition was implemented in a way that placed no restriction on the observer vector fields that label representations. By contrast, (R1-Equiv) suggests a radically different way of interpreting the Newtonian condition: it invites us to view the condition as guaranteeing the existence of a special (because twistless) set of observer vector fields, and thus also a special set of reference connections corresponding to these vector fields. Let us illustrate this point in the case of gauge field representations: (R1-Equiv) tells us that if $\nabla$ is Newtonian, we can represent $\nabla$ by means of the set of twistless representations $\{(Z, \stackrel{Z}{A})\}_{Z \in \mathcal{F}_{\text {twistless }}}$, where $\mathcal{F}_{\text {twistless }}$ denotes the set of twistless vector fields of observers. In other words, the Newtonian condition guarantees the existence of a particular 'gauge-fixing' for $\nabla$, i.e. a restriction to the set of twistless representations.

We now provide an explicit prescription for constructing these twistless representations. We will need the following proposition, which characterizes twistless vector fields:

Proposition 4.2.1. A vector field $Z^{\prime} \in \mathcal{F}$ is twistless with respect to a Newtonian connection $\nabla$ just in case it has the form

$$
Z^{\prime}=Z-h(d f),
$$

where $f$ is an arbitrary 0 -form and $Z$ is the Milne-invariant vector field defined in equation (11). 
Copyright Philosophy of Science 2017

Preprint (not copyedited or formatted)

Please use DOI when citing or quoting

Indeed, the proposition also characterizes Milne-invariant vector fields: evidently, $Z$ is itself a twistless vector field, and any $Z^{\prime}$ generated by exact 1 -form shifts will be Milne-invariant 14

Next, recall from eqn. (11) that the Milne-invariant vector field $Z$ is constructed by means of a Milne boost $N \mapsto Z$ from an arbitrary vector field of observers $N \in \mathcal{F}$, albeit one that is parameterized by the 1 -form $\stackrel{N}{A}$. Thus, this Milne boost induces a Milne symmetry transformation from an arbitrary $(N, \stackrel{N}{A})$ to a twistless $(Z, \stackrel{Z}{A})$ :

$$
N^{a} \mapsto Z^{a}=N^{a}+h^{a b}(-\stackrel{N}{A}), \quad \stackrel{N}{A} \mapsto \stackrel{Z}{A}=\stackrel{N}{A}+\stackrel{N, A}{\Phi} .
$$

Upon evaluating the result of this transformation, we find that $\stackrel{Z}{A}=\frac{1}{2} \phi t$, i.e. it is defined exclusively in terms of the clock 1-form $t$ and the Milne-invariant scalar $\phi$ (cf. eq. (10)). Furthermore, since $Z$ is Milne-invariant, this result is independent of the representative of the Milne symmetry orbit $[(N, \stackrel{N}{A})]_{\text {Milne }}$ that one chooses to apply the boost to.

We have just used the Newtonian condition to construct a twistless gauge choice, labeled by $Z \in \mathcal{F}$. However, we also know from Prop. 4.2.1 that an entire family of such choices exists, and that they are related by exact 1form shifts 15 Thus, in the parlance of physicists, the Newtonian condition only guarantees the existence of a 'partial' gauge-fixing, i.e. we are left with a residual descriptive freedom after implementing this restriction. Analyzing this residual gauge freedom now leads us to the construction of a third kind of representation for Newtonian connections.

Following BM, let us consider the pair $(Z, \phi)$, which is canonically and mutually definable from the twistless representation $\left(Z, \stackrel{Z}{A}=\frac{1}{2} \phi t\right)$. We shall call $(Z, \phi)$ a Maxwell representation of a Newtonian connection. The Maxwell representation is manifestly Milne-invariant, because both $Z$ and $\phi$ are Milneinvariant objects. However, notice that it is not invariant under the standard Maxwell symmetry action on gauge fields, i.e. $\stackrel{N}{A} \mapsto \stackrel{N}{A}+d f$, which is precisely the residual symmetry that was not 'used up' in our prescription for constructing a twistless representation. ${ }^{16}$ In fact, it is easy to check that the standard Maxwell action on the gauge fields in the definition of $Z$ induces the

\footnotetext{
${ }^{14}$ In other words, the proposition shows that the Milne-invariant vector field is unique up to exact 1-form shifts.

${ }^{15}$ We have thus at this point established the 'only if' direction of (R1-Equiv).

${ }^{16}$ Note that this Maxwell symmetry acts on gauge fields in the definition of the Milne-invariants. This should not be confused with the 'external' Maxwell action on the gauge field of $(Z, \stackrel{Z}{A})$.
} 
Copyright Philosophy of Science 2017

Preprint (not copyedited or formatted)

Please use DOI when citing or quoting

exact 1-form shifts 13 that parameterize the twistless vector fields (cf. Prop. (4.2.1)). Similarly, the Maxwell action on gauge fields induces the following transformation of $\phi$ to another Milne-invariant scalar $\phi^{\prime}$ :

$$
\phi \mapsto \phi^{\prime}=\phi+2(d f)_{a} Z^{a}-h^{a b}(d f)_{a}(d f)_{b} .
$$

By combining these transformations, we arrive at the induced Maxwell action $(Z, \phi) \mapsto\left(Z^{\prime}, \phi^{\prime}\right)$ on Maxwell representations. This action parameterizes all twistless representations of a Newtonian connection; in other words, it is precisely the descriptive freedom that remains after we have gauge-fixed to the twistless representations.

We conclude this section by noting that, just as with the previous representational strategies, Maxwell representations allow us to describe the invariant content of Newtonian connections. The relevant notion of invariance is given by the notion of a Maxwell orbit, i.e. an equivalence class $[(Z, \phi)]$ generated by the Maxwell action, whose identification with a Newtonian connection is expressed in the following proposition:

Proposition 4.2.2. The affine space of Newtonian connections is canonically isomorphic to the affine space of Maxwell orbits.

\subsection{Proto-recovery}

We now combine the resources developed in previous sections to prove a generalized recovery result that we call 'Proto-recovery'.

Proposition 4.3.1. (Proto-recovery) Let an $A$-model be the structure $(\mathcal{L}, \nabla, N)$, where $\nabla$ is Newtonian and $N \in \mathcal{F}$ is geodesic, i.e. $\stackrel{\nabla, N}{\alpha}=0$. And let a B-model be the structure $\left(\mathcal{L}, \stackrel{Z}{\nabla}, \phi_{B}, N\right)$ where $\phi_{B}$ is a scalar field, $\stackrel{Z}{\nabla}$ is a special connection (and thus Newtonian) and the acceleration of $N \in \mathcal{F}$ is $\stackrel{Z}{\nabla, N}=\frac{1}{2} h\left(d \phi_{B}\right)$. Proto-recovery then consists of two statements: (1) Given an A-model, we can recover a Maxwell orbit of B-models; indeed, the A-model can be identified with this Maxwell orbit. (2) All the models (i.e. the A model, and the B-models in the corresponding Maxwell orbit) share the same vorticity with respect to $N$.

Proof. The main idea of the proof is contained in the Milne Triangle of Figure 1, which depicts the relationships between three connections $\nabla, \stackrel{Z}{\nabla}$, and $\stackrel{N}{\nabla}$ : $\stackrel{Z}{\nabla}$ is used as a reference point to represent $\nabla$, and $\stackrel{N}{\nabla}$ is used a reference point to represent both $\nabla$ and $\stackrel{Z}{\nabla}$. The edges of the Milne Triangle represent 
Copyright Philosophy of Science 2017

Preprint (not copyedited or formatted)

Please use DOI when citing or quoting

the directional data of the representations: we decompose this data into the acceleration $\alpha$ (represented by solid arrows) and the vorticity $\omega$ (represented by dotted arrows) relative to the background inertial* structure described by the respective reference connections. The vorticity and acceleration are omitted on the left and right edges of the Milne Triangle respectively to indicate that these quantities vanish. Furthermore, we shall see that the vorticities of the bottom and right edges are the same, and that the accelerations of the bottom and left edges are the same up to a sign.

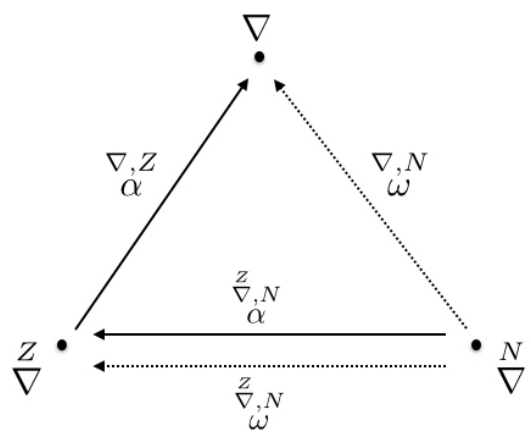

Figure 1: The Milne Triangle

We first prove part (1) of proto-recovery, viz. that the data of an A-model $(\mathcal{L}, \nabla, N)$ can be identified with a Maxwell orbit of B-models $\left[\left(\mathcal{L}, \stackrel{Z}{\nabla}, \phi_{B}, N\right)\right]$. The argument can be divided into three parts:

(1a) The Left Edge: Using $\nabla$ to construct $\left(\stackrel{Z}{\nabla}, \phi_{B}\right)$ up to Maxwell symmetry

This part of the argument follows directly from the fact (cf. Section 4.2) that any Newtonian connection $\nabla$ has a Maxwell representation $(Z, \phi)$. We then define the scalar field data $\phi_{B}$ of a B-model by identifying it with the Milne-invariant scalar $\phi$, i.e. $\phi_{B} \equiv \phi$. We have thus constructed the left edge of the Milne Triangle, and it is immediate from the considerations of Section 4.2 that this construction holds up to Maxwell symmetry.

(1b) The Right and Bottom Edges: Constructing the relationship between the $A$-model and B-model dynamics

The A-dynamics is captured by the statement that some vector field of observers $N$ is geodesic with respect to the A-connection $\nabla$. This fact can be perspicuously expressed by means of a Newton-Coriolis representation, i.e. by representing $\nabla$ through the reference data $\nabla^{N}$ and the directional data $\nabla_{\omega}^{\omega}$ and $\stackrel{\nabla, N}{\alpha}$. Since $N$ is by hypothesis geodesic with respect to $\nabla$, it is clear that the 
Copyright Philosophy of Science 2017

Preprint (not copyedited or formatted)

Please use DOI when citing or quoting

vorticity $\underset{\omega}{\nabla, N}$ is the only free directional parameter; we have thus constructed the right edge of the Milne Triangle.

We now observe two facts. First, given the Maxwell representation $(Z, \phi)$ in (1a), we know that its corresponding gauge field representation is $\left(Z, \underset{A}{A}=\frac{1}{2} \phi t\right)$, and that its corresponding Newton-Coriolis representation is $(Z, d A)$. Thus, we can readily compute the acceleration $\stackrel{\nabla, Z}{\alpha}=-\frac{1}{2} h(d \phi)$. Second, by studying the way in which acceleration transforms under the Milne boost $N \mapsto Z$, we immediately obtain the following pair of equations for the accelerations relative to $\nabla$ and $\stackrel{Z}{\nabla}, 17$

$$
\begin{aligned}
& \stackrel{\nabla, Z}{\alpha}=\stackrel{\nabla, N}{\alpha}+K \\
& \stackrel{Z}{\nabla, Z}=\stackrel{Z}{\nabla, N}+K .
\end{aligned}
$$

Since $\stackrel{Z}{\nabla, Z} \underset{\alpha}{\alpha}=0$ by the definition of a 'special connection', it follows that $\underset{\alpha}{\nabla, N}=0$ just in case $\stackrel{Z}{\nabla, N} \underset{\alpha}{\alpha}=-\stackrel{\nabla, Z}{\alpha}=\frac{1}{2} h(d \phi)$. Notice two features of this argument: (i) It is independent of the choice of $N$; (ii) it holds for any Maxwell representation $(Z, \phi)$ of $\nabla$.

At this point, we have shown that given an A-model, we can recover a B-model. We now extend this statement to the orbit of the B-model under the action of Maxwell symmetry.

(1c) Showing that an A-model can be identified with a Maxwell-orbit of $B$ models

We first stipulate that the Maxwell action on a B-model $(\mathcal{L}, \stackrel{Z}{\nabla}, \phi, N)$ is to be defined as the Maxwell action on the Maxwell representation $(Z, \phi)$ part of the B-model data. From point (ii) of $(1 \mathrm{~b})$, it is clear that this Maxwell action is compatible with the definition of a B-model, in the sense that every representative of the orbit $[(Z, \phi)]$ is also a B-model when conjoined with $N$, i.e. it satisfies the dynamical relationship described in (1b). Furthermore, it is straightforward to show that a Maxwell transformation $(Z, \phi) \mapsto\left(Z^{\prime}, \phi^{\prime}\right)$ of B-models satisfies part of the definition (eq. (5)) of a Trautman symmetry (where we set $\psi:=\phi^{\prime}-\phi$ ); however, we do not have enough data to show that it satisfies the constraint (6).

It now follows from Prop. 4.2 .2 that an A-model can be identified with its corresponding Maxwell orbit of B-models. In this sense, the dynamics of the models decouples from the standard by which we judge an A-model to be 'equivalent' to a set of B-models, i.e. the canonical identification between a

\footnotetext{
${ }^{17}$ In these equations, $K$ is the sum of tensors whose details will not be important for our purposes.
} 
Copyright Philosophy of Science 2017

Preprint (not copyedited or formatted)

Please use DOI when citing or quoting

Newtonian connection $\nabla$ and its corresponding Maxwell orbit.

(2) The Right and Bottom Edges: Showing that all models share the same vorticity

First, we show that an A-model and one of its recovered B-models share the same vorticity, i.e. we compare the dotted arrows on the right and bottom edges of the Milne Triangle. Just as in the case of acceleration in (1b), we can study how vorticity transforms under the Milne boost $N \mapsto Z$, thus obtaining the following pair of equations for the vorticities with respect to $\nabla$ and $\stackrel{Z}{\nabla}$.

$$
\begin{aligned}
& \stackrel{\nabla, Z}{\omega}=\stackrel{\nabla, N}{\omega}+d \chi \\
& \stackrel{Z}{\nabla, Z}=\stackrel{Z}{\vec{\omega}, N}+d \chi,
\end{aligned}
$$

where $\chi$ is a 1 -form parametrizing the boost. But since $\stackrel{Z}{\stackrel{Z}{\omega}, Z}=0$ by the definition of 'special connection' and $\underset{\omega}{\omega}=0$ by the fact that a Maxwell representation is a twistless gauge choice, it follows that the A-model vorticity (the dotted right edge of the triangle) is equal to the B-model vorticity (the dotted bottom edge of the triangle), i.e.

$$
\stackrel{\nabla, N}{\omega}=\stackrel{Z}{\nabla, N}=-d \chi .
$$

Notice that the argument that the A-model and the B-model share the same vorticity is independent of our choice of $N$; however, the value of their vorticity does depend on $N$.

Since our choice of B-model in the above argument was arbitrary, it follows that all B-models share the same vorticity with respect to $N$. As a consistency check, we now show that the relationship (20) is invariant under the Maxwell transformation $(Z, \phi) \mapsto\left(Z^{\prime}, \phi^{\prime}\right)$. This is so because the Maxwell boost $Z \mapsto Z^{\prime}$ induces the following relationship between vorticities:

$$
\stackrel{Z^{\prime}}{\omega}=\stackrel{N}{\omega}+d(\chi+d f)
$$

where $f$ is a 0 -form and $\chi$ is the same 1 -form as in equations 18, 19). Since $d^{2}=0$, the B-model vorticities are invariant under Maxwell transformations.

We now discuss the ways in which gauge symmetry and gauge-fixings are implicated in Proto-recovery. Two kinds of partial gauge-fixings are relevant: 
Copyright Philosophy of Science 2017

Preprint (not copyedited or formatted)

Please use DOI when citing or quoting

First, the twistless gauge-fixing that stems from the Newtonian condition is used to define the Maxwell representation data of the B-models. Thus, the Maxwell gauge symmetry acts on the left edge of the Milne triangle, thereby parametrizing the set of B-models. Second, we can view the geodesic condition on the dynamical vector field $N \in \mathcal{F}$ as a gauge-fixing condition on Newton-Coriolis representations of the A-model connection $\nabla$. Milne symmetry transformations that preserve this 'geodesic gauge' thus acts on the right edge of the triangle (while preserving the dynamical relationship between an A-model and a B-model). Since a fixed $N$ is itself part of the dynamical data of the models, this symmetry in fact parameterizes a family of Proto-Recovery results: it shows that the structure of the result is invariant under such transformations of the dynamical data (however, note that such transformations of $N$ will in general change the value of the vorticity). We thus see that the recovery result involves a subtle interplay between the Maxwell and Milne gauge symmetries acting on the left and right edges of the triangle respectively.

It is worth noting that Proto-recovery is a highly schematic result: it needs to be 'filled in' with more determinate content (e.g. source equations) before it can describe real physical scenarios. However, what we are concerned to emphasize here is the striking number of features of Trautman Recovery that can be traced to the Newtonian condition when it is interpreted as the existence of a twistless gauge-fixing: the relationship between the equations of motion (1) and (3), part (5) of the Trautman symmetry, and the non-uniqueness of recovered models all flow from this. In addition, we have the result of Part 2 , viz. that all models share the same vorticity; as we shall discuss in Section 5, this result - in conjunction with Prop. 4.2.2 - provides a generalization of recent results (Dewar, 2017; Weatherall, 2016) concerning the equivalence of Maxwell gravitation and Newton-Cartan gravitation.

The above discussion will continue to apply, mutatis mutandis, in our version of Trautman Recovery. To conclude this section, let us highlight a feature of Proto-recovery that will not carry over to Trautman Recovery: Recall that since every Newtonian connection $\nabla$ is also a special connection, i.e. $\nabla \equiv N^{\prime}$, $\nabla$ can be represented by the Maxwell representation $\left(N^{\prime}, \phi=0\right)$, where $N^{\prime}$ is not only twistless but also geodesic. Thus, supposing that $\nabla$ is the connection of an $A$-model, it is itself contained (under the description of a special connection) in the element $\left(\mathcal{L}, N^{\prime}, 0, N\right)$ of the corresponding Maxwell orbit of B-models. As we are about to see, the additional gauge-fixing of the next section will pry the represented connection apart from its orbit of recovered models. 
Copyright Philosophy of Science 2017

Preprint (not copyedited or formatted)

Please use DOI when citing or quoting

\subsection{Recovering Trautman Recovery}

We now argue that Trautman Recovery can be obtained as a special case of the Proto-recovery result from the previous section.

Proposition 4.4.1. (Recovered Trautman Recovery) (1) A Newton-Cartan model (of gravitation) $(\mathcal{L}, \nabla, \rho, N)$ can be identified with a 'Trautman orbit' $[(\mathcal{L}, \stackrel{Z}{\nabla}, \phi, \rho, N)]$ of Neo-Newtonian models (of gravitation). (2) Furthermore, all models share the same vorticity with respect to $N \in \mathcal{F}$.

Proof. Our strategy for obtaining Trautman Recovery as a special case of Proto-recovery will be to further gauge-fix the twistless (partial) gauge of Section 4.2; the result will still be a partial gauge, albeit one with even less descriptive freedom than the twistless gauge-fixing. Given that we have already made use of the vorticity and acceleration parameters in the proto-recovery result, it is reasonable to expect that the additional gauge-fixing will be controlled by the expansion parameter. As we shall see, this is indeed the case.

We begin by using the data of the Proto-recovery models to (partially) define the data of the Trautman models. Let a Newton-Cartan model be defined from the A-model data $(\mathcal{L}, \nabla, N)$ by adding to it a (non-vanishing) mass density $\rho$ and the requirement that (R2) and the source equation (4) are satisfied.

We then apply Proto-recovery to the Newton-Cartan model, thus yielding a Maxwell orbit of B-models. In conjunction with the mass density $\rho$, we will use the data $(\mathcal{L}, \stackrel{Z}{\nabla}, \phi, N)$ of a B-model to partially define a Neo-Newtonian model. In order to reproduce the standard formulation of Trautman Reovery, we will need to show that by combining this data with (R2), we can recover a Trautman orbit of Neo-Newtonian models. In other words, we will need to establish:

(a) The flatness of the connection $\stackrel{Z}{\nabla}$.

(b) The relationship between the Newton-Cartan and the Neo-Newtonian source equations, i.e. (4) and (2) respectively.

(c) The fact that the gauge symmetry acting on Neo-Newtonian models is the Trautman symmetry, i.e. it satisfies (6) in addition to (5), which was already satisfied by the B-model transformations.

We now argue that this can be accomplished by means of a further gauge-fixing (of the twistless gauge), whose existence is guaranteed by (R2).

Let $\nabla$ be the connection of a Newton-Cartan model. Since the spatial flatness of $\nabla$ follows from the source equation (4), (R2-Equiv) suggests that 
Copyright Philosophy of Science 2017

Preprint (not copyedited or formatted)

Please use DOI when citing or quoting

the further gauge-fixing should be one that restricts the Maxwell representations of a spatially flat, Newtonian $\nabla$ to a special subset that quantifies over twistless and rigid vector fields; call this the Trautman gauge. We shall call such a representation a Trautman representation and denote it by $(Z, \phi)_{T}$. Furthermore, Trautman representations carry the action of a gauge symmetry $(Z, \phi)_{T} \mapsto\left(Z^{\prime}, \phi^{\prime}\right)_{T}$ that preserves the Trautman gauge, i.e. a Maxwell symmetry $A \mapsto A^{\prime}=A+d f$ that meets a further, rigidity-preserving condition. In Prop. A.0.1 of the Appendix, we argue that this rigidity-preserving condition takes the form $\nabla^{a} \nabla^{b} f=0$.

It is easy to see that this additional gauge-fixing cannot single out the Trautman representations (within the class of Maxwell representations) by means of their directional data: this is because the directional data is exhausted by the vorticity and acceleration parameters, whereas the additional feature of the Trautman gauge is its rigidity. Thus, we can infer that the additional constraint must be imposed at the level of the reference point data $\stackrel{Z}{\nabla}$. Indeed, a standard argument confirms this by demonstrating point (a), i.e. that in conjunction with spatial flatness, imposing rigidity on $Z$ forces the special connection $\stackrel{Z}{\nabla}$ to be flat. (Notice that the flatness of the reference point data implies that $\nabla$ (which is curved) cannot be in the orbit of the Trautman representation, in contrast to the Proto-recovery case.) And given (a), another routine argument delivers (b) 18

The last item on our list, viz. (c), is to show that the Trautman gaugepreserving symmetry $(Z, \phi)_{T} \mapsto\left(Z^{\prime}, \phi^{\prime}\right)_{T}$ is what we earlier called a Trautman symmetry, i.e. a symmetry characterized by (5) and (6). Since a Maxwell symmetry already satisfies (5), it only remains to show that it obeys the constraint (6); we do so in Prop. A.0.2 of the Appendix.

Establishing points (a)-(c) shows that given a Newton-Cartan model, we can recover a corresponding Trautman orbit of Neo-Newtonian models (where the Trautman symmetry action on a Neo-Newtonian model is defined by its action on the corresponding Trautman representation). Part (1) of the proposition then follows from an analogous argument to Part (1c) of Proto-recovery; and Part (2) of the proposition immediately follows because it is a special case of Part (2) of Proto-recovery.

\footnotetext{
${ }^{18}$ We shall not rehearse these arguments here, since they do not offer any new insight into the subject of Trautman Recovery; however, we refer the interested reader to Section 4.5 of Malament (2012).
} 
Copyright Philosophy of Science 2017

Preprint (not copyedited or formatted)

Please use DOI when citing or quoting

\section{$5 \quad$ Further applications}

\section{The relationship with Maxwell gravitation}

We now comment on the relationship between our work and that of Dewar $(2017)$. Let us take a Maxwell spacetime to be the structure $\left(\mathcal{L},\left[\nabla_{\text {flat }}\right]\right)$, where $\left[\nabla_{\text {flat }}\right]$ is a 'vorticity equivalence class' of flat connections, i.e. two flat connections are equivalent just in case for any $N \in \mathcal{F}$, they share the same vorticity with respect to $N$ I9 If we adopt this terminology, then it is evident that Proto-recovery implicitly defines a generalized notion of Maxwell spacetime: Let a Proto-Maxwell spacetime be the structure $(\mathcal{L},[\nabla])$, where $[\nabla]$ is a vorticity equivalence class of Newtonian connections. (Thus, a Maxwell spacetime is an example of a Proto-Maxwell spacetime, but the latter concept is more general, because its connection representatives need not be flat.) But by the reasoning in part (2) of Proto-recovery, Newtonian connections fall into the same vorticity class just in case they are the reference data (i.e. special connections $\{\stackrel{Z}{\nabla}\}$, where $Z$ is a twistless timelike vector field) of the Maxwell representations in a Maxwell orbit. Thus, a Proto-Maxwell spacetime corresponds to a Maxwell orbit of these reference connections, and the 'vorticity class' structure of the spacetime is preserved by the corresponding Maxwell transformations.

We have just seen that a Proto-Maxwell spacetime can be obtained from an orbit of Maxwell representations by omitting the directional data (i.e. the Milne-invariant scalars) of these representations. We now consider how including this directional data allows one to 'set a gravitational model' on ProtoMaxwell spacetime.

One of the contributions of (Dewar, 2017) is that it defines a clear notion of a Maxwell model of gravitation: this is taken to be the structure $\left(\mathcal{L},\left[\nabla_{\text {flat }}\right], \rho, N\right)$, where any representative $\nabla \in\left[\nabla_{\text {flat }}\right]$ satisfies the source equation $\nabla_{a} \alpha^{a}=-4 \pi \rho$ and the equation of motion $\nabla^{[a} \alpha^{c]}=0$. While the B-models of Proto-recovery are not equipped with sources, they do give us the resources to provide a deeper explanation for these equations of motion. To see this, consider the generalization of a Maxwell model to what we shall call a ProtoMaxwell model, i.e. a structure $(\mathcal{L},[\nabla], N)$, whose equations of motion are defined as follows: For any representative $\nabla \equiv \stackrel{Z}{\nabla} \in[\nabla]$, we can use its directional data $\phi$ to compute that $\nabla^{[a} \alpha^{c]}$ equals to a sum of terms linear in $\nabla^{a} Z^{b}$, which we will take to be our Proto-Maxwell equations of motion. If we apply the further gauge-fixing of Section 4.4, i.e. by taking the twistless $Z$ to be in addition rigid (so $\nabla^{a} Z^{b}=0$ ), then we recover precisely the equations of

\footnotetext{
${ }^{19}$ Note that this definition implies the 'standard of rotation' definition given in Dewar (2017).
} 
Copyright Philosophy of Science 2017

Preprint (not copyedited or formatted)

Please use DOI when citing or quoting

motion for Maxwell gravitation.

Finally, note that Prop. 4.2.2 furnishes us with a 'proto' analog of Props. 4 and 5 of Dewar (2017): By means of the correspondence with its Maxwell orbit of B-models, a Proto-Maxwell model is equivalent to an A-model. As before, the more specific results concerning Maxwell gravitation can then be obtained by the further gauge-fixing of Section 4.4 .

2. The analogy between electromagnetism and Newton-Cartan gravitation

The following surface-level analogy between the Maxwell symmetry of electromagnetic gauge fields and the Trautman gauge symmetry of Neo-Newtonian models is often noted: Each theory has an 'invariant' formulation such that, locally, there exists a gauge symmetry orbit of empirically equivalent models corresponding to the invariant formulation.

The analogy is of course correct so far as it goes. However, the analysis of the previous sections allows us to probe more deeply the analogies and the disanalogies between the two theories. First, notice that Newton-Cartan theory has a Milne gauge symmetry group that has no analog in electromagnetism. Second, if the Newton-Cartan connection is Newtonian, then 1-form gauge fields and the Maxwell symmetry group play a role in both theories. Indeed, they have the same formal source, viz. the application of Poincaré's Lemma to the equation $d F=0$ (this, then, is the deep structural feature that gives rise to the surface-level analogy). Nonetheless, this equation has a very different interpretation in each theory: In electromagnetism it is a source-free equation of motion; whereas in Newton-Cartan theory, it is the Newtonian curvature condition, as expressed within the framework of Newton-Coriolis representations. Furthermore, the Newtonian condition is characterized by the existence of a twistless partial gauge-fixing for the Newton-Cartan connection (cf. (R1Equiv)).

This brings us to a deeper, and perhaps unexpected, analogy between electromagnetism and Newton-Cartan theory. In the former, the field strength 2-form corresponds to a Maxwell orbit of gauge-dependent representations of the field strength (i.e. 1-form gauge fields). Along the same lines, one might have thought that a Newtonian connection could only correspond to a larger Milne-Maxwell orbit of representations. However, as we explained in Section 4.2 , we can use the twistless partial gauge-fixing to construct a set of Milneinvariant representations such that a Newtonian connection corresponds to a Maxwell orbit of these representations (we thus called these the 'Maxwell representations'). At this level of structure, then, the invariant content of both theories is in 1-1 correspondence with Maxwell orbits.

A final disanalogy presents itself when we consider the level of structure required by the Trautman Recovery Theorem, i.e. where we impose (R2) in addition to the Newtonian condition. As we saw, the Maxwell symmetry is 
Copyright Philosophy of Science 2017

Preprint (not copyedited or formatted)

Please use DOI when citing or quoting

insufficient to define the Trautman gauge symmetry acting on the recovered Neo-Newtonian models: in addition, one needs a rigidity-preserving gaugefixing condition corresponding to (R2). Thus, the surface-level analogy between electromagnetism and Neo-Newtonian models belies the fact that the gauge symmetry of the latter is somewhat more constrained than the Maxwell symmetry of electromagnetism. In this regard, it is interesting to compare the Trautman gauge to a partial gauge-fixing for electromagnetism such as the 'Lorenz gauge': formally, the two are very similar (compare e.g. the rigiditypreserving condition $\nabla^{a} \nabla^{b} f=0$ to the harmonic condition for the Lorenz gauge), but the latter arises solely as a means of parameterizing the descriptive freedom, whereas in the former case, the existence of such a gauge needs to be guaranteed by the curvature conditions of the corresponding NewtonCartan model.

There is much work that remains to be done in exploring these analogies. For instance, since Newton-Cartan theory has a 'gauge field representation', can one make sense of Wilson lines for these gauge fields?And to what extent would the philosophical discussion of holonomies and the Aharonov-Bohm effect carry over to such physical quantities?

\section{Reflected recovery}

Given that a Newton-Cartan model does not geometrize away the vorticity of its recovered Neo-Newtonian models, it is worth asking if there is an analog of Trautman Recovery in which the curved model does geometrize away the vorticity of its recovered models. In fact, the Milne Triangle of Figure 1 suggests a visual strategy for obtaining such a scenario: Instead of taking the (twistless) left edge of the triangle to define the data of a recovered model and using the right and bottom edges to encode the dynamical relationships, we can reflect this assignment about the center vertical axis of the triangle so that the (geodesic) right edge defines a recovered model, and the left and bottom edges encode the dynamical relationships.

Thus, in a Reflected Proto-recovery result, an A-model is defined as $(\mathcal{L}, \nabla, Z)$ where $\nabla$ is Newtonian and the dynamical vector field $Z$ is twistless, and a Bmodel is defined as $(\mathcal{L}, \stackrel{N}{\nabla}, \stackrel{\nabla, N}{\omega}, Z)$, where $N$ is geodesic with respect to $\nabla$. It is then immediate from $16,17,18,19$ that from an A-model, we can recover a family of B-models (parametrized by Milne boosts preserving the geodesic gauge-fixing for $N$ ) each of which has the vorticity $\stackrel{N}{\nabla, Z}=-\stackrel{\nabla, N}{\omega}$, and that an A-model and its B-models share the same acceleration.

A Reflected Trautman Recovery can then be obtained by imposing rigidity on $N$ (in conjunction with the geometrized source equation for $\nabla$ ) and using the Künzle-Ehlers Recovery theorem (which assumes only the existence of a 
Copyright Philosophy of Science 2017

Preprint (not copyedited or formatted)

Please use DOI when citing or quoting

rigid vector field) to obtain the full set of equations of motion for these models. Clearly, more remains to be said about the additional curvature constraints that correspond to Reflected Recovery, as well as the (perhaps hydrodynamical) scenarios in which such a result might find application, but we leave such a task to a future work.

\section{Acknowledgments}

It is a pleasure to thank the Notre Dame Philosophy of Physics Research Group, two anonymous referees, and seminar audiences at the University of Michigan Ann-Arbor and UIC for feedback on this material. Special thanks is due to Kartik Prabhu for some very helpful conversations about NewtonCartan theory.

\section{A Proofs of various propositions}

Proposition 3.2.1: This is proved as Prop. 3.14 of Bekaert and Morand (2016). Proposition 3.3.1: This is proved as Prop. 3.20 of Bekaert and Morand (2016). Proposition 4.2.1: This is proved in Appendix C of Bekaert and Morand (2016).

Proposition 4.2.2: The proof is contained in the discussion leading up to Prop. 3.25 of Bekaert and Morand (2016). Note that the relevant model space is the vector space of principal connections, and that the relevant affine isomorphism is modeled on the identity map.

Proposition A.0.1. A Maxwell symmetry $A \mapsto A^{\prime}=A+d f$ is rigiditypreserving just in case $\nabla^{a} \nabla^{b} f=0$.

Proof. Recall that a Maxwell symmetry preserves twistlessness, and that the expansion and vorticity of a vector field $Z$ can be expressed as $\nabla^{a} Z^{b}=\omega^{a b}+$ $\theta^{a b}$. Thus, $\nabla^{a} Z^{b}=0$ in the Trautman gauge (which is both twistless and rigid). The rigidity-preserving constraint can then be obtained by studying how this $\nabla^{a} Z^{b}$ transforms under the Maxwell gauge symmetry, i.e.

$$
\nabla^{a} Z^{\prime b}=\nabla^{a} Z^{b}+\nabla^{a}\left(h^{b c}(d f)_{c}\right)
$$

and imposing the Trautman gauge condition $\nabla^{a} Z^{b}=\nabla^{a} Z^{\prime b}=0$. The rigiditypreserving constraint is thus:

$$
\nabla^{a} \nabla^{b} f=0
$$


Copyright Philosophy of Science 2017

Preprint (not copyedited or formatted)

Please use DOI when citing or quoting

Proposition A.0.2. A Trautman gauge-preserving symmetry $(Z, \phi)_{T} \mapsto\left(Z^{\prime}, \phi^{\prime}\right)_{T}$ satisfies (6).

Proof. First, we set $\psi=\phi^{\prime}-\phi$. We then compute:

$$
\begin{aligned}
\nabla^{p} \nabla^{k} \psi & =\stackrel{Z}{\nabla}^{p} \nabla^{k} \psi \\
& ={ }^{Z} \nabla^{p}\left[\left(2 \nabla^{k} \stackrel{Z}{Z}_{a} f\right) Z^{\prime a}\right] \\
& =\left(2 \nabla^{p} \nabla^{k} \nabla_{a}^{Z} f\right) Z^{\prime a}+\left(2 \nabla^{k} \nabla_{a} f\right) \nabla^{p} Z^{\prime a} \\
& =0
\end{aligned}
$$

To go from the first line to the second line, we have used (15). To go from the third line to the fourth line, we have used $\nabla^{p} Z^{\prime a}=0$ and the rigidity-preserving constraint $\nabla^{a} \nabla^{b} f=0$.

\section{References}

Bekaert, X. and K. Morand (2016). Connections and dynamical trajectories in generalised Newton-Cartan gravity I. An intrinsic view. Journal of Mathematical Physics 57(2).

Bergshoeff, E. A., J. Hartong, and J. Rosseel (2015). Torsional NewtonCartan geometry and the Schrodinger algebra. Classical and Quantum Gravity 32(13), 135017.

Dewar, N. (2017). Maxwell gravitation. forthcoming in Philosophy of Science.

Geracie, M., D. T. Son, C. Wu, and S.-F. Wu (2015). Spacetime Symmetries of the Quantum Hall Effect. Phys. Rev. D91, 045030.

Knox, E. (2014). Newtonian Spacetime Structure in Light of the Equivalence Principle. British Journal for the Philosophy of Science 65(4), 863-880.

Künzle, H. P. (1972). Galilei and lorentz structures on space-time - comparison of the corresponding geometry and physics. Ann. Inst. H. Poincare Phys. Theor. 17, 337-362.

Malament, D. (2012). Topics in the foundations of general relativity and Newtonian gravitation theory. University of Chicago Press.

Saunders, S. (2013). Rethinking Newton's Principia. Philosophy of Science $80(1), 22-48$. 
Copyright Philosophy of Science 2017

Preprint (not copyedited or formatted)

Please use DOI when citing or quoting

Wallace, D. (2016). Fundamental and emergent geometry in Newtonian physics. PhilSci Archive Preprint.

Weatherall, J. O. (2016). Maxwell-Huygens, Newton-Cartan, and SaundersKnox Space-Times. Philosophy of Science 83(1), 82-92. 